\title{
FRANCISCA BOHIGAS: MUJER CATÓLICA, DIPUTADA Y PEDAGOGA (1928-1950)
}

\author{
por ALEJANDRO CAMINO RODRÍGUEZ \\ (Universidad Autónoma de Madrid)
}

\begin{abstract}
Resumen
Francisca Bohigas ha sido vista exclusivamente como la única diputada conservadora durante la segunda república española. Sin embargo, sus intentos de transgredir y/o redefinir los roles de género asignados a las mujeres por parte de las derechas españolas, convierten a su trayectoria y su pensamiento en elementos relevantes para analizar la construcción de la diferencia sexual en los sectores conservadores españoles de la primera mitad del siglo XX.
\end{abstract}

Palabras clave: mujeres católicas; género; diferencia sexual; España; siglo XX

\begin{abstract}
Francisca Bohigas has generally been perceived and analysed as the only conservative deputy in the Spanish Second Republic. However, her persistent attempts at transgressing and/or redefining the gender roles defended by the rights-wings parties are extremely interesting. Therefore, her life and thought can be key elements for the study of the construction of sexual difference in the Spanish conservative sectors of the first half of the twentieth century.
\end{abstract}

Keywords: catholic women; gender; sexual difference; Spain; twentieth century

\section{Introducción}

Francisca de Paula Bohigas y Gavilanes fue la única diputada conservadora de la segunda república española (1931-1936) y durante la posterior dictadura franquista (1939-1975) fue la única diputada del periodo republicano que tuvo un destacado papel..$^{2}$ En el presente trabajo pretendo analizar la trayectoria y el pensamiento de

1 El presente texto se enmarca en una investigación predoctoral financiada por el Ministerio de Educación, Cultura y Deporte de España, a través del programa para la Formación del Profesorado Universitario. Referencia: FPU16/02273. Quisiera agradecer a Darina Martykánová sus comentarios sobre este texto.

2 Solo ha sido objeto de estudio monográficamente in: Juan Miguel ÁLVAREZ, "Mujeres en política durante la II República (Acción Femenina Leonesa a través de la prensa [1931-1936])", Tierras de León 120-121, León 2005, pp. 255-285; Juan Miguel ÁLVAREZ, "Francisca Bohigas, una diputada de derechas en la Segunda República", Historia 16 359, Madrid 2006, pp. 88-103; María de la Concepción MARCOS DEL OLMO, "Francisca Bohigas, militancia política y actividad parlamentaria en las Cortes del segundo bienio republicano", in: María de la Concepción MARCOS DEL OLMO - Rafael SERRANO (coords.), Mujer y política en la España contemporánea (1868-1936), Valladolid 2012, pp. 203-222. 
esta mujer durante la segunda república, la guerra civil (1936-1939) y la primera década de la dictadura franquista por dos motivos principalmente:

En primer lugar, debido a que representa un buen ejemplo de las contradicciones a las que tuvieron que enfrentarse las mujeres conservadoras durante este periodo de tantos y acelerados cambios en la historia de España. La mayor incoherencia de todas sería el hecho de que estas mujeres políticas estaban al servicio de una ideología que defendía que el ámbito natural de las españolas era el hogar. Y, en segundo lugar, porque la figura de Francisca ofrece un marco privilegiado para analizar las posibilidades de acción individual que tuvieron las mujeres conservadoras dentro de los modelos hegemónicos y/o oficiales de género (que tenían modelos subalternos en competición con ellos, sobre todo durante la segunda república). Estos se presentaban como determinantes, pero en realidad sólo eran condicionantes pese a sus imposiciones porque sus contradicciones internas generaban espacios de incertidumbre que permitían crear márgenes de acción y expresión, los cuales provocaban que las pautas colectivas pudiesen adaptarse o transformarse. ${ }^{3}$ Entonces estos márgenes permitían que una persona generase prácticas, que se pueden definir como de negociación o de resistencia, con el fin de transgredir y/o redefinir el modelo de género hegemónico. En consecuencia, las católicas españolas no fueron víctimas pasivas de un orden desigual, sino sujetos activos capaces de negociar el peso de las normativas de género (aunque a veces las interiorizasen en el pensamiento y en su actuación práctica) con el fin de intentar dar solución a los desafíos existentes en el periodo para las mujeres de su ideología.

En relación con esto, un nivel de análisis clave de la presente investigación es la constante interrelación entre el discurso de género oficial de cada momento en España con la acción práctica de Francisca Bohigas, debido a que la biografía y la perspectiva de género se complementan para, partiendo de las normativas oficiales y/o hegemónicas, analizar la acción práctica de las mujeres. Este tipo de análisis proporciona muchos beneficios al combinar los enfoques históricos sociales y culturales. ${ }^{4}$ Las categorías analíticas principales que vertebran el presente análisis son la diferencia sexual, el género y las identidades. Como el sexo y el género no son esencialistas, ahistóricos o universales, el género lo entiendo como un concepto útil para el análisis histórico debido a que es la mejor herramienta posible para el estudio de la diferencia sexual, es decir, de cómo los significados de los cuerpos sexuados se producen cultural y socialmente en relación el uno con el otro, y cómo estas definiciones se modifican espacial y temporalmente. Por tanto, no pondré la atención sólo en los roles que Francisca Bohigas asignaba a las mujeres y a los hombres, sino también en como intentaba construir la diferencia sexual. En cuanto a la noción de identidad(es), defiendo fundamentalmente que

3 Barbara TAYLOR, "Separations of Soul: Solitude, Biography, History", American Historical Review 114, Chicago 2009, pp. 640-651; Mónica BOLUFER, "Multitudes del yo: biografía e historia de las mujeres", Ayer 93, Madrid 2014, p. 112.

4 Judith WALKOWITZ, City of Dreadful Delight: Narratives of Sexual Danger in Late-Victorian London, Londres 1992; Kathleen CANNING, Gender History in Practice. Historical Perspectives on Bodies, Class and Citizenship, Ithaca-Londres 2006. 
todas las personas tienen siempre adscripciones múltiples e inestables y, en este caso, que la identidad como mujeres de las políticas católicas es fundamental para entender su implicación política y social. ${ }^{5}$

\section{Contexto histórico previo a la guerra civil}

En España la exclusión de las mujeres de los derechos de la ciudadanía fue parte del proyecto liberal imperante durante el siglo XIX y las primeras décadas del $\mathrm{XX}$, pese a que eran unos derechos declarados como universales. Pero como en muchos lugares del mundo, en España durante las primeras décadas del siglo XX se transformaron los ideales de masculinidad y feminidad en diversos sentidos, de acuerdo con el carácter plural de la modernidad y de la jerarquización de las relaciones de poder en diferentes ámbitos. Tras la primera guerra mundial, la revalorización de la maternidad fue el punto clave para la creación de un renovado modelo de género que sustituía el concepto de jerarquía de sexos por el de la diferencia y complementariedad entre mujeres y hombres, aunque esto no provocaba la total desaparición de la jerarquía. Aunque este ideal continuaba defendiendo que el deber esencial de las mujeres en la vida era el ser madres, como este discurso, que se ganó en España el favor de la mayoría de los sectores sociales, ya no se efectuó desde la premisa de la teórica incapacidad de las mujeres para cumplir otro tipo de función, hubo para las españolas mejores posibilidades discursivas en el terreno laboral y en el educativo. ${ }^{6}$

Mientras, en el ámbito católico la movilización de las mujeres fue progresivamente en aumento durante el primer tercio del siglo XX. Aunque dicha movilización estuvo instrumentalizada por la Iglesia para enfrentarse a las medidas laicizadoras de los gobiernos liberales, también generó para las católicas una oportunidad de desarrollar una versión particular de la ciudadanía política femenina. Las ideas que utilizaron para legitimar su presencia pública y su reclamación de la ciudadanía política fueron las de que las mujeres tenían mayor religiosidad, poseían superioridad moral, habían resistido mejor que los hombres las embestidas de las corrientes materialistas, revolucionarias y extranjerizantes y, especialmente, la de que debían proyectar las cualidades maternas fuera del hogar para regenerar una esfera pública y política que se percibía como necesitada de atención inmediata. ${ }^{7}$

Pero no hay que perder de vista que el ideal de género católico y conservador estaba en pugna con los modelos defendidos por los republicanos liberales y por los socialistas, así como con la figura de la mujer moderna. En resumen, en estas décadas hubo diversos modelos normativos en pugna, algunos de los cuales apelaban (al menos teóricamente) al igualitarismo, mientras que otros mantuvieron

5 Sobre el género y su utilidad como herramienta analítica: Joan SCOTT, "Género: ¿Todavía una categoría útil para el análisis?”, La manzana de la discordia 6, Cali 2011, pp. 95-101. Sobre la noción de identidad: Joan SCOTT, "El eco de la fantasía: la historia y la construcción de la identidad", Ayer 62, Madrid 2006, pp. 111-138.

6 Nerea ARESTI, Médicos, donjuanes y mujeres modernas. Los ideales de feminidad y masculinidad en el primer tercio del siglo XX, Guipúzcoa 2001, pp. 257-259.

7 Inmaculada BLASCO, "Citizenship and Female Catholic Militancy in 1920s Spain", Gender \& History 3, Glasgow 2007, pp. 441-466. 
explícitamente concepciones que se fundamentaban en la diferencia entre los sexos. Estas variaciones se debieron a la pervivencia de los viejos modelos de género y a la emergencia de otros nuevos, así como al carácter diversificado de la modernidad y de las relaciones sociales entre ambos sexos. Posteriormente contra toda esta incertidumbre de género buscará reaccionar el régimen franquista, tanto discursivamente como con el arma en la mano.

\section{La formación de Francisca Bohigas y sus primeros pasos en la política}

Francisca Bohigas nació en Barcelona en 1892 en el seno de una familia profundamente religiosa. Pese a que no era una familia perteneciente a las capas más adineradas de la sociedad, sus padres le apoyaron y le facilitaron el acceso a un nivel educativo del que pocas mujeres (y hombres) podían disfrutar en España entonces $\mathrm{y}$ fomentaron que tuviese una trayectoria profesional.

La biografiada consiguió diversos títulos académicos de enseñanza superior, entre los que resalta su licenciatura en Filosofía y Letras por la Universidad Central de Madrid. Destaca también que fuese miembro becada de la Residencia de Señoritas y que recibiese una pensión en 1924 por la Junta para la Ampliación de Estudios e Investigaciones Científicas (JAE) para realizar estudios en Suiza y Alemania de psicología aplicados a la educación. Por tanto, perteneció al reducidísimo número de mujeres relacionadas con dos de las instituciones más importantes para el desarrollo del capital humano investigador en España durante el primer tercio del siglo XX: la primera fue un centro que posibilitó que las mujeres de las clases acomodadas de fuera de Madrid pudiesen acudir a la capital española a cursar estudios superiores, mientras que la segunda fue una institución liberal que abogó por tratar de incluir en España los métodos pedagógicos y teorías científicas más avanzadas de Europa. ${ }^{8}$

Toda esta trayectoria, sumada a que llegó a formar parte de la junta directiva de una organización feminista como fue la Juventud Universitaria Feminista, hace que sea necesario indicar que durante la juventud su toma de conciencia, como ciudadana y como mujer, no la desarrolló en círculos católicos sino en organizaciones de corte progresista. Esto tiene la explicación de que, pese a que durante las primeras décadas del siglo XX en España se transformasen los ideales de masculinidad y de feminidad en diversos sentidos, el que se mantuviese el discurso de la domesticidad como fundamento ideológico del modelo de género predominante condicionó que

8 Archivo General de la Universidad Complutense de Madrid (en adelante sólo AGUCM), TIT, 200, 26; Expediente JAE/22-401; Archivo General de la Administración (en adelante sólo AGA), (05)001.019, caja 31/17421, exp. 1955-25; AGA, (05)020.000, caja 32/15932, exp. 9744-10; Fundación José Ortega y Gasset, Fondo de la Residencia de Señoritas, Serie de Correspondencia, 25/68/1, Carta de Francisca Bohigas a María de Maeztu en agosto de 1916; Memoria de la JAE. Memoria correspondiente a los años 1916 y 1917, pp. 258-265; Memoria de la JAE. Memoria correspondiente a los años 1918 y 1919, pp. 302-303; Memoria de la JAE. Memoria correspondiente a los cursos 1924-1925 y 1925-1926, pp. 25-26. En los estudios sobre la Residencia de Señoritas suele omitirse su presencia. La excepción más importante: Paloma ALCALÁ, "Francisca Bohigas Gavilanes", in: Paloma ALCALÁ - Capi CORRALES - Julia LÓPEZ (coords.), Ni tontas ni locas: las intelectuales en el Madrid del primer tercio del siglo XX, Madrid 2009, pp. 147-148. 
los diferentes feminismos españoles elaborasen un discurso que tomase como base para la justificación de sus demandas la diferencia de género. Esto tuvo la consecuencia de que los planteamientos de las feministas católicas no se diferenciasen demasiado (ni en forma ni en contenido) de las propuestas de las feministas socialistas de la época. ${ }^{9}$

Para el estudio de cómo los diferentes feminismos españoles se retroalimentaron mutuamente es especialmente útil la biografía, ya que puede ayudar a comprender mejor el tipo de relaciones que establecieron las feministas de una y otra vertiente. Al pertenecer la mayoría a la reducida elite intelectual del país, muchas de ellas compartieron espacios en las universidades españolas, en las asociaciones universitarias, en la Residencia de Señoritas, etc., y desarrollaron su concienciación feminista a la vez que aprendían a actuar colectivamente en aquellos espacios públicos en los que se permitía su presencia. ${ }^{10}$

Mientras todo esto ocurría, Francisca comenzó en 1920 su trayectoria profesional al conseguir una plaza como inspectora de primera enseñanza en Lleida, el cual era entonces un ámbito profesional que permanecía fuertemente masculinizado. Sin embargo, su importante actividad política no empezó hasta que a comienzos de 1928 fue destinada a León. ${ }^{11}$ En este territorio, uno de los más conservadores de España, alcanzó rápidamente un fuerte protagonismo en la vida pública y política. De hecho, en el marco de unos actos de propaganda que desarrolló en León a finales del verano de 1928 la Unión Patriótica (UP), el partido creado por el entonces dictador Miguel Primo de Rivera, se convirtió en la primera mujer que en la provincia leonesa tomaba parte de un mitin. Pero no era un orador más: cuando el periódico conservador más importante de la provincia, el Diario de León, publicitó el acto central de esta campaña, sólo indicó que en el mismo tomaría parte Francisca Bohigas, pese a que en el mitin también intervino, entre otros, el alcalde de la ciudad. ${ }^{12}$

9 Mary NASH, "Experiencia y aprendizaje: la formación histórica de los feminismos en España", Historia social 40, Valencia 1994, pp. 161-162; Cristina ESCRIVÀ - Julia JORDÀ - José Vicente MARTÍ, "Elisa Soriano Fischer, puntal del feminismo moderno: Presidenta de la juventud universitaria feminista (1920-1928)", in: Pilar FOLGUERA et al. (eds.), Pensar con la Historia desde el siglo XXI. XII Congreso de la Asociación de Historia Contemporánea, Madrid 2015, pp. 965-985.

10 Sólo así se puede comprender la amistad que mantuvieron en los años de juventud la socialista Margarita Nelken y Francisca Bohigas: Heraldo de Madrid, 4 de mayo de 1934, p. 14; Heraldo de Madrid, 24 de marzo de 1936, p. 16. La mejor muestra de la amistad es que Nelken le dedicó un libro suyo: Margarita NELKEN, La condición social de la mujer de la mujer en España, Madrid 1975 [1919]. Aunque habitualmente se ha dudado de si la Paquita Bohigas de la dedicatoria era la biografiada en el presente texto, puedo afirmar con rotundidad que sí lo era. De todas formas, esta no es la única muestra de que Francisca fue una mujer de ideas socialistas en su juventud: Centro Documental para la Memoria Histórica (en adelante sólo CDMH), PS. Madrid. 449/57, Expediente de la causa abierta contra García García, Onofre, 13 de septiembre de 1935.

11 AGA, (05)001.019, caja 31/17421, exp. 1955-25; AGA, (05)001.010, legajo 16696 Top. 33/71, exp. 12 y Gaceta de Madrid, 13 de febrero de 1928, p. 1075.

12 Diario de León, 9 de septiembre de 1928, p. 8 y Mujeres Españolas, 17 de noviembre de 1929, p. 14. La capacidad de la inspectora para invisibilizar, o relegar a una segunda fila, a los hombres que iban a compartir tribuna con ella permanecerá a lo largo de su trayectoria política. Por ejemplo: Diario de León, 24 de marzo de 1933, p. 8; Diario de León, 13 de julio de 1933, p. 7; Heraldo de Madrid, 2 de agosto de 1935, p. 7. 
Este vertiginoso ascenso político solo puede explicarse porque los dirigentes conservadores leoneses pronto vieron en su figura a una herramienta clave para atraer a las mujeres a sus posicionamientos ideológicos, debido a que durante la dictadura de Primo de Rivera se produjo el primer intento estatal de incluir a las mujeres en la política formal (aunque sólo mediante una legislación limitada de derechos políticos). Asimismo, no se puede olvidar que la biografiada en estas fechas ya era una de las referencias del movimiento pedagógico católico, tanto a nivel nacional como regional. Este movimiento pedagógico tenía el objetivo fundamental de reaccionar ante las tendencias educativas innovadoras que llegaban de Europa y EEUU, especialmente por las ideas que conllevaban de coeducación o de neutralidad religiosa en las escuelas. ${ }^{13}$

Desde 1929 y hasta la proclamación de la segunda república, siguió participando con gran asiduidad como elemento destacado en los actos que organizaban los sectores sociales vinculados a su ideología. Pero lo más destacado que hizo fue proponerse, mediante una serie de conferencias y de artículos, visibilizar la labor que las leonesas estaban desplegando en el hogar y en la esfera pública y el convertirse en la primera mujer que en León realizase un esfuerzo serio para organizar y movilizar a las leonesas en torno a los ideales de la derecha católica y alrededor de la mejora en la situación de las mujeres. ${ }^{14}$

Con el fin de conseguir estos objetivos, aunque nunca reivindicó la igualdad completa entre hombres y mujeres en todos los ámbitos, siempre defendió la idea de que era necesario que las mujeres se implicasen activamente en la vida política y pública. En su opinión eso tendría muchos beneficios para la sociedad debido a que por sus características naturales específicas (como la intuición) podrían resolver una serie de problemas trascendentales que los hombres eran incapaces de solucionar. Además, reivindicó constantemente que las españolas estaban igual de capacitadas para intervenir en política que los hombres: “¿Qué las mujeres se ocupan de cosas que no entienden? Ya las aprenderán. También los hombres las aprendieron y muchos de ellos todavía no las saben [...]". ${ }^{15}$

Esto se debe a que las cualidades diferenciadas que, según ella, poseían de forma innata y natural las personas de cada sexo no implicaban gradación de superioridad o inferioridad; de hecho, implícitamente otorgaba mayor importancia a las virtudes propias de las mujeres. Estas ideas tenían una fuerte similitud a las defendidas por las máximas representantes del feminismo católico. Este feminismo se puede definir como una propuesta de ciudadanía femenina católica debido a que sus creadoras y difusoras propagaron una concepción de las mujeres como sujetos políticos

$13 A B C, 6$ de noviembre de 1928, p. 25; El Distrito Universitario, 29 de noviembre de 1928, p. 3; El magisterio español, 26 de febrero de 1929, p. 615; Diario de León, 18 de octubre de 1929, p. 7; ABC, 31 de diciembre de 1929, p. 28.

14 Diario de León, 11 de febrero de 1929, p. 1; Diario de León, 27 de abril de 1929, p. 9; Diario de León, 5 de julio de 1929, p. 1; Diario de León, 10 de diciembre de 1929, p 1; Diario de León, 14 de marzo de 1930 , p. 1.

15 Diario de León, 10 de abril de 1930, p. 1. 
y públicos, lo cual difuminaba el ideal de las esferas separadas que defendía el modelo de género liberal decimonónico. ${ }^{16}$

Aunque Francisca Bohigas nunca se definió como feminista católica (pese a reivindicar frecuentemente demandas más transgresoras y potencialmente emancipadoras que esta corriente), queda claro que la propia identidad de la biografiada como mujer es fundamental para entender su implicación política y pública y sus intentos de redefinir las ideas hegemónicas de las derechas españolas respecto a la diferencia sexual. Por ejemplo, realizó insistentemente una defensa implícita en favor del empoderamiento de las mujeres, al reivindicar que era necesario que las españolas confiasen más en sus capacidades y se hiciesen conscientes de que todas tenían talento. Asimismo, defendió que, si las mujeres tenían menor nivel intelectual y cultural que los varones se debía a que habían recibido menor formación, no tenían los mismos derechos laborales y estaban en inferioridad ante la ley. En consecuencia, pidió a las españolas que luchasen para modificar una situación que les hacía portadoras de las mismas obligaciones que los hombres, pero de menos derechos. ${ }^{17}$ Por último, reivindicó siempre la necesidad de que las mujeres tuviesen las mismas oportunidades que los hombres a la hora de formarse para el trabajo asalariado que deseasen, debido a que así tendrían la posibilidad de ganarse la vida por sí solas sin depender de nadie:

Y si conseguimos algún día que la mujer en lugar de confiar la solución de su vida al matrimonio, cuente con un arte, oficio o carrera, que le permita ganarse [...] la vida cuando le sea preciso también irá al matrimonio, pero irá por afecto, por interés moral, por deseo de crear una familia, y sin apremio económico viéndose libre del deber de enamorarse del primero que le dirija la palabra. Y habremos ganado en libertad y en moralidad. ${ }^{18}$

De todas formas, aunque sus propuestas fuesen en ocasiones transgresoras respecto al modelo de mujer que proponía el grueso del movimiento católico femenino, es conveniente destacar que todo este pensamiento no estaba aislado de su contexto social, ya que estaba influido por su militancia en la Agrupación Nacional de Mujeres Españolas, cuyo ideario apuntaló una separación en las formas de concebir la acción sociopolítica de las conservadoras. Por un lado, Acción Católica de la Mujer basándose en la maternidad social pedía que las españolas desarrollasen sus virtudes naturales fuera del hogar, pero siempre concibiendo esta actuación

16 Para un excelente análisis teórico sobre la utilización del concepto de feminismo católico: Inmaculada BLASCO, "Definir y explicar el feminismo histórico. Debates y propuestas de análisis desde la historia", in: Ángela Cenarro - Régine Illion (coords.), Feminismos: contribuciones desde la historia, Zaragoza 2014, pp. 267-289. Para el pensamiento de la biografiada: Francisca BOHIGAS, Actuación española en América. (Resumen escolar), Madrid 1930, pp. 260-263; Diario de León, 27 de agosto de 1928, p. 3; Diario de León, 10 de septiembre de 1928, p. 2; Mujeres Españolas, 14 de abril de 1929, pp. 11-12; Mujeres españolas, 27 de abril de 1930, p. 11; Mujeres españolas, 11 de mayo de 1930, p. 9.

17 Mujeres españolas, 4 de mayo de 1930, p. 11; Mujeres españolas, 14 de septiembre de 1930, p. 4.

18 Diario de León, 24 de septiembre de 1929, p. 1. 
como algo temporal y como un deber; no como un derecho. Mientras, por otro lado, las feministas católicas de la Agrupación Nacional de Mujeres Españolas, aunque también partían de la defensa de la maternidad social para legitimar el acceso de las mujeres a la esfera pública, incluyeron en su discurso la reivindicación de la defensa de la igualdad de derechos jurídico-políticos entre las mujeres y los hombres y la idea de que las españolas tenían el derecho (y el deber) de participar permanentemente en la esfera pública para solucionar lo que los hombres no habían conseguido arreglar. ${ }^{19}$

Sin embargo, los años 1930 y 1931 fueron un periodo difícil para Francisca Bohigas. Cuando cayó la dictadura de Primo de Rivera, la corona de Alfonso XIII, que había avalado la dictadura, se quedó tambaleando. Entonces, la inspectora, que se autodefinía como monárquica y católica, trató de encabezar en León una campaña en favor de la monarquía. De todas formas, pese a que fueron momentos complejos para las opciones políticas monárquicas y católicas, no tuvo inconveniente en criticar públicamente con cierta dureza a la mayoría de los oradores de estos posicionamientos ideológicos por su incapacidad para analizar los problemas familiares, ya que consideraba que por las virtudes propias de las mujeres ellas eran las únicas con la capacidad suficiente para interpretarlos correctamente. ${ }^{20}$

\section{La segunda república española: el periodo de mayor implicación política}

Con la llegada de la segunda república, como ocurrió en buena parte de España, en León quedó desarticulada la derecha católica, siendo Francisca Bohigas una de las personas que más trabajaron para reorganizarla. Estas actuaciones provocaron que los sectores republicanos y socialistas le acusasen de deslealtad al régimen republicano y tratasen de conseguir que fuese destinada profesionalmente a otra provincia. Ante estas acusaciones, la inspectora de primera enseñanza buscó defenderse y zanjar la situación declarando su "leal adhesión a la República". ${ }^{21}$

Más allá de esta polémica, como ya era una figura importante consolidada dentro de la derecha leonesa, fue tentada por Acción Nacional (AN), el partido conservador más importante a nivel nacional, para que formase parte de la candidatura que iba a concurrir por León a los comicios generales de junio de 1931, pero Francisca Bohigas rechazó la propuesta debido a la desorganización de la formación política. De todas formas, finalmente AN no fue a la lucha electoral en esa circunscripción,

19 La Vanguardia, 16 de mayo de 1930, p. 8; Rebeca ARCE, La construcción social de la mujer por el catolicismo y las derechas españolas en la época contemporánea, Tesis doctoral, Cantabria 2016, pp. 211-214. Como la doctrina católica convertía a la maternidad en la función esencial de las mujeres, la maternidad social permitía que las mujeres que no eran madres biológicas cumpliesen de otra manera su misión maternal: mediante obras de caridad, como pilares de la recatolización de España, etc. Esto convertía a las católicas en elementos fundamentales de la lucha contra los considerados males que asolaban a España, lo cual estimulaba (aunque de forma más limitada que otros feminismos) sus crecientes aspiraciones de participar en la esfera pública.

20 Diario de León, 7 de marzo de 1930, p. 1 y Diario de León, 3 de junio de 1930, p. 1.

${ }_{21}$ CDMH, PS Madrid 423; Diario de León, 16 de mayo de 1931, p. 4. 
lo cual dejó el camino libre para el aplastante triunfo en la provincia de los republicanos y de las izquierdas..$^{22}$

Como en la mayoría de España, los sectores conservadores leoneses aprendieron que debían reorganizarse si querían revertir en el futuro los resultados de estos comicios. Con este objetivo, desde finales de 1931 la biografiada se convirtió en una figura central en la reconstrucción de las derechas leonesas: lideró la creación de Acción Femenina Leonesa (AFL) y se convirtió en su presidenta. Esta organización nació con los objetivos de convertirse en una agrupación política de masas que aglutinase a las mujeres conservadoras de todas las clases sociales y de fomentar que las leonesas utilizasen los nuevos derechos políticos que poseían. ${ }^{23}$

Durante los siguientes meses, la líder de AFL argumentó constantemente que era necesario que las mujeres se implicasen en los asuntos políticos y se integrasen, en función de su ideología, en cualquier asociación que les sirviese a modo de escuela de ciudadanía. Y es que su retórica estuvo cargada de un intachable respeto por la pluralidad democrática durante todo el periodo republicano, algo de lo que no podían presumir demasiados/as políticos/as entonces; de hecho, no tuvo inconveniente en posar en el Parlamento junto al busto de Mendizábal, sobre quién recaía el mito de ser el máximo representante del anticlericalismo desde que desamortizó los bienes del clero regular. ${ }^{24}$ En palabras de Francisca Bohigas:

Mi objeto, por hoy, es ilustrar a las mujeres que no lo estén aún [...] acerca de los derechos que recientemente nos ha concedido la ley; [...] toda mujer que haya cumplido los veintitrés años tiene el derecho y el deber de emitir su voto en las futuras elecciones [...]. Agrupaos [...]. Pero antes de agruparos pensad bien en qué partido os vais a afiliar $[\ldots]$.

Yo os aconsejaría que os afiliaseis en Acción Femenina Leonesa [...]; leed su argumento, estudiadlo, pensadlo bien [...], y si están conformes con vuestros sentimientos, inscribíos en sus listas, si sus teorías no os satisfacen, agrupaos a otro partido que os guste más; pero asociaos, solas, no iréis a ninguna parte; y cuando llegue el día de las elecciones emitid vuestro sufragio [...]. Si triunfásemos, sepamos ser generosos con los vencidos; si nos derrotasen, tengamos serenidad y sigamos trabajando sin desmayo para conseguir la victoria en otra ocasión. ${ }^{25}$

En este tiempo tampoco dejó en segundo plano la lucha por empoderar a las españolas. Siguiendo el discurso de los años anteriores, mantuvo que estaban tan (o tan poco) capacitadas como los hombres para la actuación pública. Pero ahora, también partiendo desde la diferencia sexual que defendía el movimiento católico

22 Pablo VILLALAÍN, Mujer y política, la participación de la mujer en las elecciones generales celebradas en Madrid durante la II república (1931-1936), Madrid 2000, p. 125.

23 Diario de León, 2 de enero de 1932, pp. 1-4; Región, 27 de febrero de 1932, p. 16.

24 Diario de León, 4 de enero de 1932, pp. 2-3; Diario de León, 19 de enero de 1932, p. 8 y Diario de León, 4 de julio de 1932. Sobre Mendizábal: AGA, Archivo fotográfico Alfonso. f-botigas-001.jpg; Juan Luis PAN-MONTOJO, "Juan Álvarez y Mendizábal (1790-1853): el burgués revolucionario", in: Isabel Burdiel - Manuel Pérez Ledesma (coords.), Liberales, agitadores y conspiradores: biografías heterodoxas del siglo XIX, Madrid 2000, pp. 155-182.

25 Diario de León, 20 de febrero de 1932, p. 8. 
femenino, otorgó a las mujeres unas capacidades supuestamente naturales que favorecían que sufragasen mejor que los hombres. Estos planteamientos, pese a que siempre pidió a las mujeres que combinasen sus deberes y derechos políticos con la labor en el hogar, no dan pie a interpretar que simplemente trataba de organizarles por mero interés electoral (que era lo único que pretendía en estas fechas buena parte del movimiento católico) ${ }^{26} \mathrm{Como}$ el prestigio político de la inspectora a nivel nacional siguió creciendo progresivamente, desde comienzos de 1932 comenzó a ser considerada por muchos analistas como una de las mujeres más importantes del panorama político conservador español. De hecho, pese a que fue sospechosa de haber estado implicada en la trama del golpe de Estado contra la república liderado por Sanjurjo, en octubre de 1932 fue una de las delegadas con mayor protagonismo de la asamblea nacional en la que AN pasó a denominarse Acción Popular (AP). ${ }^{27}$

Poco después, como era el elemento básico de la estrategia de la derecha provincial para ganar adeptos entre las mujeres, fue la principal encargada de intentar orientar los votos de las españolas hacia las derechas en las elecciones municipales parciales de abril de 1933. Tanto fue así que Francisca fue la más activa propagandista conservadora de la provincia. En el periodo de campaña pidió constantemente a sus oyentes varones que respetasen la ideología y el voto de sus esposas, y a éstas que votasen masivamente en función sus propios ideales. Sin embargo, la inspectora fue más agresiva que previamente con el resto de las opciones políticas debido a la tensión que imperó durante la campaña, a la creciente radicalización que hubo durante esos meses en las filas de la AP y a la necesidad de aglutinar el voto del electorado conservador porque el ámbito político derechista estaba muy fragmentado. ${ }^{28}$ Finalmente, en estas elecciones las derechas salieron vencedoras, aunque el triunfo fue lógico porque se celebraron en zonas de fuerte tradición conservadora. Para Francisca Bohigas estos comicios demostraron que las españolas favorecían con sus votos sobre todo a las opciones políticas conservadoras, lo cual en su opinión se debía a que las mujeres eran más religiosas que los hombres y estaban más preocupadas por defender a la familia, al catolicismo y a la patria. ${ }^{29}$

Francisca ya era claramente la mujer más importante de AP a nivel nacional, lo cual quedó constatado cuando, en marzo de 1933, fue una de las personas elegidas para intervenir en los mítines que clausurarían el congreso nacional de AP y que servirían para presentar el surgimiento de una nueva organización política: la Confederación Española de Derechas Autónomas (CEDA). Asimismo, al quedar los órganos rectores de la CEDA dominados por los elementos moderados, Francisca fue elegida, junto con Abilia Arroyo, para ocupar en el consejo nacional el cargo de

26 Diario de León, 28 de junio de 1932, p. 3; El Día de Palencia, 5 de julio de 1932, p. 6; El correo de Zamora, 14 de marzo de 1933, p. 2.

27 La Gaceta de Tenerife, 8 de octubre de 1932, p. 6; Diario de León, 20 de abril de 1933, p. 2; Diario de León, 12 de septiembre de 1932, p. 6.

28 Diario de León, 20 de febrero de 1933, p. 8; Diario de León, 15 de abril de 1933, p. 3; El correo de Zamora, 17 de abril de 1933, pp. 1-3; Diario de León, 19 de abril de 1933, p. 4.

29 Diario de León, 2 de mayo de 1933, p. 1; La Gaceta de Tenerife, 27 de mayo de 1933, p. 1. 
vocal representante de las agrupaciones femeninas.$^{30}$ Pero fue tras este congreso el momento en el que su importancia y prestigio dentro de los sectores conservadores llegó a su máximo esplendor, cuando los medios de comunicación afines tergiversaron un pequeño problema profesional que tuvo la inspectora para representarla como una mártir que era perseguida por el gobierno republicano debido a sus ideas políticas. ${ }^{31}$

En el discurso que desarrolló Francisca Bohigas en los meses posteriores, aunque las ideas fundamentales en las que incidió fueron prácticamente las mismas que anteriormente, también hubo elementos novedosos ya que probablemente se vio en la necesidad de adaptar su discurso al de la CEDA y al del movimiento católico femenino (que cada vez tenía un carácter menos transgresor). La más destacada de las nuevas ideas fue la de que si las españolas comenzaron a intervenir masivamente en la vida pública, dejando en un segundo plano al hogar y a la familia, se debió a que se vieron en la necesidad de luchar contra las leyes laicas implantadas por el gobierno republicano por el gran mal que, en su opinión, podían causar al país. ${ }^{32}$

Posteriormente la biografiada fue elegida para concurrir a las elecciones generales de noviembre y diciembre de 1933 en la candidatura de la CEDA por León, secundando al líder de esta, José María Gil Robles. Durante esta campaña ante todo destaca que siguiese defendiendo la necesidad de que las mujeres votasen masivamente según sus ideales, independientemente de la orientación de sus votos. Por tanto, a diferencia del resto de líderes y candidatos del panorama católico y conservador español (no solo de la CEDA), Francisca no confirió al voto de las mujeres gran importancia sólo por el oportunismo político de que podría beneficiar a las derechas, sino porque era un derecho democrático básico para su desarrollo como personas: ${ }^{33}$

Votad gentes de vuestra ideología, pero votad. Invocando el artículo 27 de la Constitución, que proclama la libertad de conciencia pid[o] a las izquierdas que dejen en libertad a la mujer para ejercitar su voto, a las derechas, se lo pid[o] además en nombre de un deber de conciencia. ${ }^{34}$

En la primera vuelta de las elecciones la inspectora consiguió hacerse con un escaño por la provincia leonesa después de que la CEDA venciese ampliamente; pero fue la menos votada de la candidatura pese a que si se omite a Gil Robles era quién

30 José Ramón MONTERO, La CEDA. El catolicismo social y político en la II República, Madrid 1977, p. 508 y p. 683; Javier TUSELL, Historia de la democracia cristiana en España I. Los antecedentes. La CEDA y la II República, Madrid 1974, p. 194; ABC, 1 de marzo de 1933, p. 28; ABC, 5 de marzo de 1933, p. 2.

31 Gaceta de Madrid, 11 de marzo 1933, p. 1905; AGA, (05)001.010, legajo 16696 Top. 33/71, exp. 12; Región, 12 de marzo de 1933, p. 8; Igualdad, 13 de marzo de 1933, p. 3; El Diario Palentino, 14 de marzo de 1933, p. 1; La Cruz, 15 de marzo de 1933, p. 1; El correo de Zamora, 20 de marzo de 1933, p. 1; ABC, 21 de marzo de 1933, p. 36; Acción, 23 de marzo de 1933, p. 1.

32 Igualdad, 2 de octubre de 1933, p. 3; El correo de Zamora, 2 de octubre de 1933, p. 3.

33 Diario de León, 2 de noviembre de 1933, p. 2.

34 Diario de León, 17 de abril de 1933, p. 1. 
tenía mayor relevancia política en la provincia. Esto se debió a que a Francisca Bohigas le perjudicó su condición de mujer en las elecciones, aunque al conjunto de candidatas no les perjudicó claramente su sexo una vez incluidas en las candidaturas porque los/as españoles/as apoyaron mayoritariamente a los candidatos ideológicamente más cercanos sin importarles este aspecto. De hecho, si hubo pocas mujeres electas (sólo cinco, cuatro socialistas más la biografiada) se debió a que la mayoría de las candidatas se presentaron por circunscripciones en las que su partido tenía escasas opciones de obtener escaños. ${ }^{35}$

Sin embargo, desafortunadamente para las españolas de la ideología de la nueva diputada, estas elecciones supusieron un punto y aparte en el movimiento católico de mujeres. Nada más producirse los comicios, los líderes conservadores y la prensa afín reaccionaron ante los planteamientos transgresores en materia de género que estaban desarrollando muchos sectores de mujeres conservadoras y frente a la cada vez mayor movilización política de estas mujeres. Para estos sectores una cosa era la movilización electoral de las mujeres para obtener réditos en las urnas, pero otra muy diferente el que se dedicasen exclusivamente a la actividad política. Por este motivo las derechas reivindicaron que las españolas debían centrar sus esfuerzos en cumplir con las funciones domésticas que les eran asignadas como naturales por razón de su sexo. La situación descrita implicó a la mayoría de las feministas católicas, quienes implícitamente se vieron en la necesidad de abandonar el discurso sobre la ciudadanía femenina, en beneficio del de la necesidad de defender la familia y la religión frente a la supuesta amenaza laicista y el desorden social. De todas formas, aunque Francisca Bohigas se vio afectada por este viraje general y los años como diputada fueron el periodo de su vida en el que defendió un ideal de mujer menos transgresor, no tuvo problema en criticar a su partido por no haber incluido a más mujeres en las candidaturas y por no haber recompensado políticamente la gran labor realizada en la campaña electoral por las conservadoras. Asimismo, continuó reivindicando que las españolas debían seguir implicadas en la esfera política porque, como se habían visto menos afectadas que los hombres por las teorías materialistas y laicistas, su labor en la campaña y sus votos siempre serían decisivos para que venciesen las derechas en las urnas. ${ }^{36}$

En las Cortes la inspectora desplegó una importante actividad parlamentaria. ${ }^{37}$ Sin embargo, como consecuencia de lo que acabo de exponer, desde que obtuvo el acta de diputada sus intervenciones públicas se centraron fundamentalmente en abordar aspectos relacionados con la educación. Siguiendo las directrices indicadas en la Encíclica Divini Illius Magistri de Pío XI sobre la educación cristiana de la

35 Alejandro CAMINO, "La influencia de las mujeres españolas en los resultados de las elecciones generales de 1933”, Revista Historia Autónoma 11, Madrid 2017, pp. 179-197.

36 Ellas, 10 de diciembre de 1933, p. 1; Acción, 22 de diciembre de 1933, p. 1; Diario de León, 21 de mayo de 1935, p. 3; El correo de Zamora, 16 de septiembre de 1935, p. 4; ABC, 17 de septiembre de 1935, p. 35 .

37 Diario de Sesiones del Congreso de los Diputados (en adelante sólo DSCD), 11 de enero de 1934, p. 449; DSCD, 9 de octubre de 1934, pp. 4489-4490; DSCD, 19 de diciembre de 1934, p. 5639; La Vanguardia, 22 de marzo de 1935, p. 22; DSCD, 6 de diciembre de 1935, p. 11195; ABC, 31 de diciembre de 1935 , p. 18. 
juventud (1929), se centró en la necesidad de modificar lo que ella consideraba como planes educativos laicos y desespañolizados.

En especial, Francisca Bohigas fue profundamente contraria a la coeducación, al considerar que hombres y mujeres tenían por naturaleza una función social distinta que debía estimularse en centros adaptados y al interpretar que la coeducación provocaba desordenes en las atribuciones de género tal y como ella las concebía. Este pensamiento estuvo influido por la postura vaticana y fue compartido por la mayoría del movimiento católico en España. Incluso, a finales de 1934 presentó al Congreso una proposición de ley que buscaba acabar con la coeducación en los centros de formación de maestras y maestros, la cual, aunque nunca llegó a aprobarse, generó un fuerte debate público. ${ }^{38}$

Pero también realizó muchas reivindicaciones fundamentalmente corporativas con el fin de mejorar la situación de los profesionales de la educación. De hecho, compartió muchas de las medidas implantadas por el gobierno del primer bienio republicano con el fin de profesionalizar la labor de los/as docentes y de los/as inspectores. Toda esta actividad ha provocado que algunos/as autores no duden en defender que fue una de las personas con mayor influencia dentro de la política educativa durante la segunda república. ${ }^{39}$

Cuando las Cortes fueron disueltas y se convocaron nuevas elecciones generales para febrero de 1936, Francisca Bohigas intentó concurrir de nuevo a los comicios, pero la CEDA decidió no incluirla en la candidatura por León (por lo que ese partido no presentó a ninguna candidata en toda España). Esto fue consecuencia de su enfrentamiento directo con el líder regional de la CEDA y con el propio Gil Robles, quienes se vengaron de la inspectora por haber cuestionado constantemente su liderazgo en la organización. Esta situación fue el desencadenante para que Francisca se decidiese a abandonar la CEDA y la AFL. ${ }^{40}$

Para cerrar este apartado creo que es interesante destacar la imagen que se proyectó en la prensa de Francisca Bohigas desde finales de la dictadura de Primo de Rivera hasta que perdió su escaño en el Parlamento. En los medios afines siempre se la presentó como una mujer de grandes capacidades intelectuales, oradoras y de liderazgo, que además había comprendido adecuadamente en qué consistía el feminismo, a diferencia de las mujeres de izquierda. También frecuentemente se la representó como una persona ejemplar por su respeto a las opiniones ajenas y como una mujer valiente que no tenía inconveniente en enfrentarse dialécticamente a los hombres contrarios a su ideología. No en vano, la biografiada no rehuía el

38 Esta idea no era nueva en ella: Diario de León, 27 de abril de 1929, p. 9. Para su proposición de ley: DSCD, 5 de febrero de 1935, pp. 6064-6065.

39 Helio CARPINTERO - Fania HERRERO, "La Junta para ampliación de estudios y el desarrollo de la psicología española”, Asclepio. Revista de Historia de la Medicina y de la Ciencia 2, Madrid 2007, p. 206. Algunos ejemplos de las reivindicaciones: Heraldo de Madrid, 5 de junio de 1934, p. 5; DSCD, 26 de junio de 1934, pp. 4015-4020; El Siglo futuro, 13 de marzo de 1935, p. 6; DSCD, 29 de mayo de 1935, pp. 7798-7804; DSCD, 27 de junio de 1935, pp. 8634-8636.

40 Diario de León, 16 de enero de 1936, p. 11; La Libertad, 1 de febrero de 1936, p. 1; ABC, 28 de mayo de 1936, p. 21. 
enfrentamiento con nadie y sacó a relucir a menudo su fuerte carácter. ${ }^{41}$ De hecho, era una mujer que se autovaloraba como alguien tan importante que, en el Parlamento, era "el único diputado de la Ceda que cuando habla el Sr. Gil Robles no se cree en la necesidad de que la vea sonreír o aprobar cuanto dice [...]". ${ }^{42}$

\section{La comprometida posición de Francisca Bohigas durante guerra civil y la necesidad de adaptarse a los nuevos tiempos durante el franquismo}

Tras las elecciones de febrero de 1936 hasta el comienzo de la guerra civil, la inspectora desapareció de la primera plana de la escena pública y política leonesa, por lo que apenas hay constancia de sus actuaciones en este periodo. ${ }^{43} \mathrm{Sin}$ embargo, se le han atribuido dos tipos de trayectorias muy diferentes. Por un lado, algunos/as autores defienden que se integró en la extrema derecha del Bloque Nacional, posiblemente influidos por el testimonio de Gil Robles. Aunque no hay que descartar la posibilidad, como la relación del líder de la CEDA y la inspectora no era mala, es posible que distorsionara la realidad con una intención interesada. Mientras, otros/ as autores han defendido que antes de la guerra civil la biografiada desarrolló una actividad en favor de Manuel Portela Valladares, queriendo mostrar que su abandonó de la CEDA se debió a que decidió apostar por una solución política centrista para los problemas del país. ${ }^{44}$

Francisca Bohigas sufrió muchos sobresaltos durante la guerra civil (la mayoría de los miembros del cuerpo de inspectores de primera enseñanza fueron represaliados por motivos políticos e ideológicos). Nada más comenzar la guerra el gobierno republicano la cesó por considerarla como enemigo del régimen. Sin embargo, como León pasó rápidamente a ser controlado por los militares sublevados contra el gobierno democrático de la república, no sólo se mantuvo en su puesto, sino que además se convirtió en la Inspectora Jefe de la provincia. ${ }^{45}$

Las evidencias de la simpatía de Francisca Bohigas por la causa sublevada son muchas. Pero la mejor prueba se produjo cuando a finales de agosto publicó un manifiesto en el que afirmó que era necesario que todos los leoneses apoyasen al ejército sublevado que luchaba para salvar al país y a la civilización cristiana. Sin

41 Un buen ejemplo es que no tuviese problema en enfrentarse al director del Diario de León: Gerardo LEÓN, Conservadurismo en Castilla. La trayectoria política de Ricardo Cortes Villasana. $1905-$ 1936, Tesis Doctoral, Valladolid 2015, p. 262. Sobre las caracterizaciones en la prensa: Diario de León, 27 de noviembre de 1928, p. 1; Mujeres Españolas, 17 de noviembre de 1929, p. 14; Diario de León, 19 de enero de 1932, p. 8; Diario de León, 8 de marzo de 1932, pp. 1-5; Diario de León, 23 de marzo de 1932, p. 7; El Día de Palencia, 13 de mayo de 1932, p. 1; El correo de Zamora, 14 de marzo de 1933, p. 2; Diario de León, 21 de marzo de 1933, p. 1; El correo de Zamora, 17 de abril de 1933, pp. 1-3; La Prensa, 8 de febrero de 1934, p. 3.

42 El Sol, 28 de febrero de 1935, p. 8.

43 Con algunas excepciones: La Vanguardia, 12 de junio de 1936, p. 18; La Vanguardia, 2 de julio de 1936, p. 16.

44 Esperanza GARCÍA, La actuación de la mujer en las Cortes de la II República, Madrid 1979, p. 48; VILLALAÍN, Mujer, p. 411; José María GIL ROBLES, No fue posible la paz, Barcelona 1968 , pp. 615-616; Wenceslao ÁLVAREZ, La represión de postguerra en León. Depuración de la enseñanza (1936-1943), León 1986, p. 48.

45 La Libertad, 4 de agosto de 1936, p. 6; Diario de León, 21 de agosto de 1936, p. 4. 
embargo, pese al apoyo al bando sublevado poco después fue destituida de su cargo y suspendida de empleo y sueldo. Como su depuración levantó ciertas suspicacias, los responsables de esta tuvieron que justificar los motivos de su actuación. Su explicación se centró en indicar que no les quedó más remedio puesto que Francisca se encontraba en paradero desconocido debido a que se estaba escondiendo de un "movimiento popular de animadversión hacia ella". Aunque esta situación existió no se puede saber si realmente fue lo que provocó su cese. De todas formas, muy pronto se le levantó la sanción y, nada más terminar la guerra, se le nombró Inspectora de primera enseñanza de Madrid con carácter provisional. ${ }^{46}$

Francisca Bohigas desde la inmediata postguerra fue una de las personas que más contribuyó, desde varios cargos institucionales (fue vicedirectora del Museo Pedagógico Nacional y profesora-auxiliar en la Sección de Pedagogía de la Universidad Central de Madrid), a desarrollar el tejido de la pedagogía española en el franquismo. Además, la exdiputada también colaboró en este proceso con su obra La inspección de primera enseñanza y la educación de la generación nueva (1941), que fue el más destacado intento durante la inmediata postguerra de diseñar un nuevo modelo de inspección de primera enseñanza, y con sus artículos en Consigna. Revista Pedagógica de la Sección Femenina y en Revista de pedagogía española. Sin embargo, durante el franquismo su pensamiento en materia educativa no sufrió grandes cambios en relación con las décadas anteriores. De hecho, defendió en sus planteamientos continuidades respecto al modelo educativo reformista implantado durante el primer bienio republicano, aunque incorporó la idea de que para devolver a España su grandeza histórica era necesario un modelo educativo totalmente alejado de los principios liberales. ${ }^{47}$

Durante los primeros lustros del franquismo Francisca mantuvo, con altibajos, un fuerte protagonismo en la esfera pública, sobre todo porque le correspondió frecuentemente presidir actos oficiales e institucionales una vez que se convirtió en 1950 en inspectora central de enseñanza primaria y, un año y medio después, en inspectora extraordinaria permanente. Además, siguió impartiendo conferencias sobre cuestiones pedagógicas y sobre la situación de las mujeres en España en múltiples congresos, ya que todavía era valorada como una destacadísima personalidad en estas materias. ${ }^{48}$

46 Archivo Regional de la Comunidad de Madrid. Fondo documental de la Diputación. Expediente de depuración de Rosa Bohigas Gavilanes. Signatura 2/15; AGA, (05)001.010, legajo 16696 Top. 33/71, exp. 12; Diario de León, 11 de agosto de 1936, p. 2; Boletín oficial de la provincia de León, 29 de agosto de 1936, pp. 3-4; Boletín oficial de la provincia de León, 15 de octubre de 1936; BOE, 21 de noviembre de 1936, pp. 232-233; Atenas, diciembre de 1936, pp. 408-409; Boletín oficial del Estado (en adelante sólo BOE), 6 de marzo de 1937, p. 610.

47 AGUCM, P-0744, 38; Francisca BOHIGAS, La inspección de primera enseñanza y la educación de la generación nueva, Madrid 1941, pp. 5-10, 55 y 97; Mujeres españolas, 27 de abril de 1930, p. 11; Consigna, abril de 1941, pp. 11-12; Luis BATANAZ, La Inspección de Primera Enseñanza durante la guerra civil y el primer franquismo (1936-1943). Represalia y desgarramiento, Córdoba 2011, p. 64; Juan MAINER, La forja de un campo profesional. Pedagogía y didáctica de las ciencias sociales en España (1900-1970), Madrid 2009, p. 523.

48 BOE, 5 de septiembre de 1950, p. 3894, BOE, 1 de febrero de 1952, p. 475; Escuela Española, 14 de febrero de 1952, p. 106; Falange. Eco de Canarias, 21 de septiembre de 1952, p. 10; La Vanguardia, 
También cabe destacar que no parece que la ex diputada estuviese nunca muy implicada con la Sección Femenina de Falange (SF), la organización femenina "oficial" del régimen, pese a que fue su asesora pedagógica. Esto se debe a que ella proveía del movimiento católico y no del falangismo, por lo que durante el franquismo fue más cercana a los sectores católicos del régimen que a los círculos falangistas. Sin embargo, hubo un momento de su relación con la SF que es necesario destacar. Durante la Junta Consultiva de la SF de 1957 Francisca apoyó la idea de una de sus compañeras de que para que la SF consiguiese atraer a las mujeres al régimen, era necesario primero que Franco definiese de forma más clara la política nacional. Esto suponía, en última instancia, elevar la responsabilidad de que no tuviesen mayores éxitos al propio dictador. Esta es una prueba más de que las jerarquías falangistas no cumplían con la abnegación y la sumisión de las mujeres a los hombres que tanto predicaban. ${ }^{49}$

De todas formas, lo más destacado de todo es que durante el franquismo Francisca Bohigas también realizó una prolífica labor de difusión de ideas. Colaboró constantemente con revistas y publicó dos manuales de conducta: Hogar (1941) y ¿Qué profesión elegir? Guía de profesiones femeninas (1947). En ellos, aunque en teoría trató de colaborar en el asentamiento del modelo oficial de género que el franquismo pretendía convertir en hegemónico, como suele ocurrir cuando alguien pretende transmitir unas normas oficialistas, en realidad las adaptó a su pensamiento y trató de redefinir lo que debía ser una mujer socialmente aceptable..$^{50}$

Para poder analizar esto correctamente es necesario introducir en qué consistía el modelo oficial de género que el franquismo pretendió imponer. Desde el principio el franquismo intentó poner fin a la incertidumbre de género existente durante las décadas anteriores, por lo que buscó diseñar un modelo de género basado en el determinismo biológico y en las ideas de la complementariedad y la diferencia (con jerarquización), con el objetivo de que garantizase las diferencias sexuales y el orden social establecido. Los elementos claves del modelo de mujer ideal que defendió el franquismo en estos años fueron el matrimonio y la maternidad (que fue entendida como un acto de reproducción biológica, social y cultural), los cuales se presentaban como los destinos ideales y naturales para la mayoría de las españolas. Por último, hay que tener presente que el discurso de género franquista no se diferenció demasiado del propuesto por los regímenes fascistas contemporáneos. ${ }^{51}$

21 de agosto de 1960, p. 6; AGA, (05)001.010, legajo 16696 Top. 33/71, exp. 12. Sobre los diversos congresos: Archivo de la Residencia de Estudiantes. Signatura 41194/28; La Vanguardia, 20 de octubre de 1949, p. 4; Revista de Pedagogía Española, octubre de 1949, pp. 95-103; Imperio, 20 de diciembre de 1949, p. 2; Lanza, 22 de mayo de 1951, p. 3.

49 Luis SUÁREZ, Crónica de la Sección Femenina y su tiempo, Madrid 1992, pp. 323-325. Para uno de los ejemplos más significativos de la falta de sumisión: Antonio MORANT I ARIÑO, "Para influir en la vida del estado futuro': discurso y práctica falangista sobre el papel de la mujer y la feminidad, 1933-1945", Historia y política: Ideas, procesos y movimientos sociales 27, Madrid 2012, pp. 136-138.

50 Para un análisis pormenorizado de sus manuales de formación: Alejandro CAMINO, "Entre el hogar y la profesión: los manuales de conducta de Francisca Bohigas durante el primer franquismo (1939. 1950)", Travessias 12 (1), Paraná 2018, pp. 224-242.

51 Carme MOLINERO RUÍZ, "Mujer, franquismo, fascismo”, Historia Social 30, Valencia 1998, pp. 97-117. 
En el análisis del pensamiento de Francisca Bohigas durante los años cuarenta, que abordo a continuación, mostraré los ejes fundamentales de su retórica y sus contradicciones, partiendo de la base de que en estos años propuso un ideal de mujer cercano en muchos aspectos a los planteamientos oficialistas del franquismo, pero también alejado en muchos otros. ${ }^{52}$

Para la biografiada las españolas, en tanto que madres, esposas e hijas, debían cumplir en el hogar su destino biológico (encomendado por Dios desde los orígenes). Si no desempeñaban esta función, que Francisca naturalizaba, podrían provocar terribles males a su familia y a España. ${ }^{53}$ Sin embargo, ella misma contradecía en su acción práctica estas ideas porque nunca estuvo casada y debido a que tampoco destinó, a juzgar por su intensa actividad pública, muchos esfuerzos al cuidado de su propio hogar.

También, como había hecho a lo largo de su vida, defendió que las mujeres contaban con una serie de características naturales que eran diferentes a las de los hombres. En su opinión estas virtudes, en especial la intuición, otorgaban a las españolas un papel vital en la construcción del nuevo orden porque, al ser las principales encargadas de la educación de sus hijos y al tener la capacidad de estimular a los hombres, podían dotar a todas las personas españolas de las herramientas necesarias para regenerar el país. ${ }^{54}$

Aunque las propuestas señaladas en los últimos párrafos tenían mucha cercanía al modelo de mujer deseado por el franquismo, la biografiada difirió públicamente de muchos aspectos del ideal propuesto por el régimen y la SF. Esto en buena parte se debió a que, como provenía del pensamiento católico, no se sentía cerca del ideario falangista. Por ejemplo, pese a que trató de regular las relaciones dentro del noviazgo, nunca abordó el tema de la virginidad (algo que hicieron muchos otros teóricos), y fue una de las pocas personas que se preocuparon por normativizar la niñez, ya que como el matrimonio y la maternidad quedaron configurados en el discurso oficial franquista como los objetivos ideales para las mujeres, la infancia y la vejez no solían ser focos de interés para estos teóricos. También hubo claras divergencias con el ideal de mujer franquista cuando defendió que las españolas debían ser totalmente dueñas de sí mismas, por lo que tenía que ser respetado si una mujer se quedaba soltera de forma voluntaria. Por lo tanto, si bien la soltería no era lo ideal, valoraba que debía ser socialmente aceptable y respetable (lo cual era tradicional en la cultura católica). Esto se diferenciaba de la tan recurrente apelación a la escasez de varones provocada por la guerra que utilizaban las jerarquías de la SF para justificar su soltería. ${ }^{55}$

De todas formas, es al analizar el discurso sobre el trabajo asalariado de las españolas cuando mejor se aprecian las diferencias entre el ideal de mujer que proponía

52 Por este motivo es un tanto inadecuada la afirmación de que mediante sus artículos se pueden conocer las propuestas generales de la SF y del régimen en estas materias: Elena POSA, "Actituds al voltant de la coeducació”, Papers: revista de sociología 9, Barcelona 1978, pp. 66-69.

53 Francisca BOHIGAS, Hogar, Madrid 1941, p. 3; Consigna, abril de 1943, pp. 20-23; Revista de Pedagogía Española, junio de 1948, p. 19.

54 Por tanto, siguió preocupándose de definir la diferencia sexual: Consigna, agosto de 1941, p. 10.

55 BOHIGAS, Hogar, pp. 118-122; BOHIGAS, La inspección, pp. 33-34. 
el franquismo y el que defendía Francisca Bohigas. Aunque tuvo muchas contradicciones por querer respetar en apariencia las estructuras patriarcales, esta precursora de los estudios de orientación profesional en España buscó redefinir el ideal de mujer socialmente aceptable. Con esta intención, la exdiputada reivindicó constantemente que, debido a las pésimas circunstancias económicas, como la mayoría de hombres no podían satisfacer con su salario las necesidades del hogar, al menos a corto y medio plazo era necesario que las mujeres trabajasen asalariadamente para completar ingresos. Aunque defendía que lo ideal era que las necesidades materiales las resolviese en exclusiva el varón cabeza de familia (para respetar la estructura patriarcal del régimen), trasladaba Bohigas esta posibilidad a un futuro lejano e hipotético: ${ }^{56}$

Las niñas, ¿deben prepararse para el oficio natural de ser madres solamente? ¿Deben unir a esa preparación la técnica de una profesión que les permita ganar su sustento y ayudar al marido? [...].

La experiencia que nos envuelve demuestra que son millares las mujeres que tienen que ganarse la vida. Solteras, casadas y viudas. Hoy, esa imperiosa necesidad existe para la mujer con independencia del estado civil [...]. Por esta razón, es necedad manifiesta hablar de la NECESIDAD DE REINTEGRAR LA MADRE AL HOGAR $[\ldots]$.

La única manera de que la mujer pueda reintegrarse al hogar y deje de ser competidora del hombre en la lucha por la vida, es hacer al hombre capaz de subvenir las necesidades mínimas de un hogar. ${ }^{57}$

También la biografiada expuso siempre que las mujeres debían elegir estudiar y aprender una profesión que fuese, en la medida de lo posible, compatible con sus obligaciones familiares y sus responsabilidades en el hogar. Sin embargo, si bien es cierto que consideraba que las españolas por sus virtudes naturales se ajustaban mejor a las profesiones relacionadas con la enseñanza o la medicina, ya desde antes del franquismo reivindicaba que eran válidas para ejercer todo tipo de trabajos, incluso aquellos que mayoritariamente estuviesen desempeñados por hombres. Por este motivo rechazaba, más inconsciente que conscientemente, la idea de la mayoría de los teóricos durante el franquismo de que había una serie de profesiones naturales para las personas de cada sexo. ${ }^{58}$ Además, sobre un tema que le había venido preocupando y que ahora vuelve a plantear constantemente, reivindicó que era necesario que las españolas aprendiesen un oficio para que así pudiesen contemplar su futuro con seguridad, sin verse forzadas "a un matrimonio de conveniencia ni a convertirse en una carga para los suyos". ${ }^{59}$ Todo esto era transgresor en un contexto en el que el

56 Consigna, noviembre de 1941, p. 12; Revista de Pedagogía Española, junio de 1948, pp. 17-19.

57 BOHIGAS, Hogar, pp. 106-107. Las mayúsculas aparecen en el escrito original de Bohigas.

58 Francisca BOHIGAS, ¿Qué profesión elegir? (guía de profesiones femeninas), Madrid 1947; Mujeres españolas, 17 de agosto de 1930, p. 4; Mujeres españolas, 28 de septiembre de 1930, p. 4; Consigna, noviembre de 1945, pp. 15-18; Consigna, junio de 1946, pp. 15-18; Consigna, octubre de 1946, p. 15; Revista de Pedagogía Española, abril de 1947, pp. 17-19; Consigna, enero de 1953, p. 25.

59 BOHIGAS, ¿Qué profesión elegir?, p. 5. 
Estado franquista buscó apartar a las mujeres, en especial a las casadas, del trabajo remunerado.

Sin embargo, la exdiputada tenía un modelo de hombre ideal parecido al propuesto por los teóricos franquistas. Siguiendo las doctrinas eclesiásticas defendió que los varones debían ser siempre la cabeza de familia y la máxima autoridad en el hogar, por lo que, en consecuencia, las mujeres debían estar sometidas a las decisiones de su marido. También defendió que esa estructura familiar debía mantenerse, aunque las mujeres trabajasen asalariadamente, para que en ningún caso los hombres españoles viesen atacada su masculinidad. Pero, a diferencia de lo propuesto por el modelo oficial franquista, como defendía que en el matrimonio ambos debían respetarse mutuamente y que las mujeres no debían asumir una docilidad total, en cierto modo mantenía una postura ambigua:

El marido es jefe económico del hogar y quien tiene la obligación de hacer frente a todas las necesidades económicas, lógicas del mismo, y la mujer viene obligada a su debida administración; pero cuando la insuficiencia económica del marido es manifiesta, esposa e hijos tienen la obligación moral de ayudarlo sin quebrantar su autoridad. ${ }^{60}$

De todas formas, esto lo hacía principalmente para no quebrantar en apariencia el orden patriarcal, ya que llegó a defender que los maridos debían implicarse en ayudar a las esposas en la educación de sus hijos. Este matiz es importante porque normalmente los manuales de formación dedicados a establecer el ideal masculino en estos años no hacían este tipo de referencias. ${ }^{61}$

\section{Conclusiones}

El análisis de la figura de Francisca Bohigas, como ha quedado demostrado, proporciona un marco privilegiado para analizar las posibilidades de acción individual que tuvieron las españolas conservadoras dentro de unos modelos hegemónicos y oficiales de género (cambiantes a lo largo del periodo estudiado), que se presentaban como determinantes, pero que en realidad solo eran condicionantes.

La biografiada, que a lo largo de toda su vida realizó reivindicaciones específicas que buscaban una mejora de la situación de las mujeres en España, buscó redefinir el modelo de mujer socialmente aceptable que trataban de imponer los modelos de género oficialistas de cada momento. En consecuencia, las propuestas normativas críticas con los modelos oficiales que siempre realizó favorecen el conocimiento general de los desafíos que en estas décadas tuvieron las españolas de su ideología y, además, permiten analizar las contradicciones de la movilización política de las mujeres católicas y conservadoras en España.

Asimismo, se puede afirmar que Francisca Bohigas, como muchas otras mujeres del movimiento católico español que mantuvieron siempre a la religión católica en

60 BOHIGAS, Hogar, p. 20.

61 Ibidem, pp. 59-60; Consigna, mayo de 1941, p. 9; Consigna, septiembre de 1941, p. 12; Consigna, febrero de 1943, pp. 19-21. 
el centro mismo de su identidad política y de género, tuvo a lo largo de su vida un pensamiento que se puede calificar como feminista, ya que si bien estaba basado en un modelo de género convencional (lo cual limitaba los márgenes de actuación), ponía claramente en cuestión la ideología de la domesticidad y la exclusión de las mujeres de la esfera pública.

De todas formas, el modelo de feminidad alternativo que propuso Francisca Bohigas trascendió en ocasiones al feminismo católico y, por supuesto, a la maternidad social. La biografiada no sólo buscó transgredir y redefinir el modelo ideal de mujer hegemónico, sino que buscó construir la diferencia sexual de una manera que, especialmente en el ámbito político, era tendente a la igualdad entre sexos. Por tanto, si bien otorgó a las españolas una serie de virtudes naturales que justificasen su papel en la esfera pública, Francisca no se conformó con esto y reivindicó la total igualdad de derechos políticos para las personas de los dos sexos, sin ningún tipo de matiz, y el deber y el derecho de que las mujeres participasen en la esfera política y en la vida pública. Aunque reivindicaba que las españolas podían realizar todo esto sin abandonar o descuidar sus deberes supuestamente naturales en el hogar y para con la familia.

(Escrito en español por el autor)

\section{BIBLIOGRAFÍA}

ALCALÁ, Paloma, "Francisca Bohigas Gavilanes", in: Paloma Alcalá, Capi Corrales y Julia López (coords.), Ni tontas ni locas: las intelectuales en el Madrid del primer tercio del siglo XX, Madrid: FECYT, 2009, pp. 147-148.

ÁLVAREZ, Juan Miguel, "Mujeres en política durante la II República (Acción Femenina Leonesa a través de la prensa [1931-1936])", Tierras de León 120-121, León, 2005, pp. 255-285.

ÁLVAREZ, Juan Miguel, "Francisca Bohigas, una diputada de derechas en la Segunda República", Historia 16 359, Madrid, Historia e Información, 2006, pp. 88-103.

ÁLVAREZ, Wenceslao, La represión de postguerra en León. Depuración de la enseñanza (1936-1943), León: Batalla de Clavijo, 1986.

ARCE, Rebeca, La construcción social de la mujer por el catolicismo y las derechas españolas en la época contemporánea, Tesis doctoral, Cantabria: Universidad de Cantabria, 2016.

ARESTI, Nerea, Médicos, Donjuanes y Mujeres Modernas. Los ideales de feminidad y masculinidad en el primer tercio del siglo XX, Guipúzcoa: Editorial de la Universidad del País Vasco, 2001.

BATANAZ, Luis, La Inspección de Primera Enseñanza durante la guerra civil y el primer franquismo (1936-1943). Represalia y desgarramiento, Córdoba: Servicio de Publicaciones de la Universidad de Córdoba, 2011.

BLASCO, Inmaculada, "Citizenship and Female Catholic Militancy in 1920s Spain”, in: Gender \& History 3, Glasgow: Wiley, 2007, pp. 441-466.

BLASCO, Inmaculada, "Definir y explicar el feminismo histórico. Debates y propuestas de análisis desde la historia", in: Ángela Cenarro y Régine Illion (coords.), Feminismos: contribuciones desde la historia, Zaragoza: Prensas de la Universidad, 2014, pp. 267-289.

BOHIGAS, Francisca, Actuación española en América. (Resumen escolar), Madrid: Biblioteca Española Femenina, 1930.

BOHIGAS, Francisca, La inspección de primera enseñanza y la educación de la generación nueva, Madrid: Editorial Cigüeña, 1941. 
BOHIGAS, Francisca, Hogar, Madrid: Gráficas Reunidas, 1941.

BOHIGAS, Francisca, ¿Qué profesión elegir? : guía de profesiones femeninas, Madrid: Mayfe, 1947.

BOLUFER, Mónica, "Multitudes del yo: biografía e historia de las mujeres", Ayer 93, Madrid, Marcial Pons, 2014, pp. 85-116.

CAMINO, Alejandro, "La influencia de las mujeres españolas en los resultados de las elecciones generales de 1933", Revista Historia Autónoma 11, Madrid, 2017, pp. 179-197.

CAMINO, Alejandro, "Entre el hogar y la profesión: los manuales de conducta de Francisca Bohigas durante el primer franquismo (1939-1950)", Travessias 12 (1), Paraná: Unioeste, 2018, pp. 224-242.

CANNING, Kathleen, Gender History in Practice. Historical Perspectives on Bodies, Class and Citizenship, Ithaca-Londres: Cornell University Press, 2006.

CARPINTERO, Helio y HERRERO, Fania, "La Junta para ampliación de estudios y el desarrollo de la psicología española", Asclepio. Revista de Historia de la Medicina y de la Ciencia 2, Madrid, CSIC, 2007, pp. 181-212.

ESCRIVÂ, Cristina - JORDÀ, Julia - MARTí, José Vicente, "Elisa Soriano Fischer, puntal del feminismo moderno: Presidenta de la juventud universitaria feminista (1920-1928)", in: Pilar Folguera et al. (eds.), Pensar con la Historia desde el siglo XXI. XII Congreso de la Asociación de Historia Contemporánea, Madrid: UAM ediciones, 2015, pp. 965-985.

GARCÍA, Esperanza, La actuación de la mujer en las Cortes de la II República, Madrid: Ministerio de Cultura, Almena, 1979.

GIL ROBLES, José María, No fue posible la paz, Barcelona: Ariel, 1968.

LEÓN, Gerardo, Conservadurismo en Castilla. La trayectoria política de Ricardo Cortes Villasana. 1905-1936, Tesis Doctoral, Valladolid: Universidad de Valladolid, 2015.

MAINER, Juan, La forja de un campo profesional. Pedagogía y didáctica de las ciencias sociales en España (1900-1970), Madrid: CSIC, 2009.

MARCOS DEL OLMO, María de la Concepción, "Francisca Bohigas, militancia política y actividad parlamentaria en las Cortes del segundo bienio republicano", in: María de la Concepción Marcos del Olmo y Rafael Serrano (coords.), Mujer y política en la España contemporánea (1868-1936), Valladolid: Universidad de Valladolid, 2012, pp. 203-222.

MOLINERO RUÍZ, Carme, "Mujer, franquismo, fascismo", Historia Social 30, Valencia, Fundación Instituto de Historia Social, 1998, pp. 97-117.

MONTERO, José Ramón, La CEDA. El catolicismo social y político en la II República, Madrid: Ediciones de la Revista de Trabajo, 1977.

MORANT I ARIÑO, Antonio, “'Para influir en la vida del estado futuro': discurso y práctica falangista sobre el papel de la mujer y la feminidad, 1933-1945", Historia y política: Ideas, procesos y movimientos sociales 27, Madrid: Centro de Estudios Políticos e Institucionales, 2012, pp. 113-141.

NASH, Mary, "Experiencia y aprendizaje: la formación histórica de los feminismos en España", Historia social 40, Valencia, Fundación Instituto de Historia Social, 1994, pp. 151-172.

NELKEN, Margarita, La condición social de la mujer de la mujer en España, Madrid: CVS, 1975 [1919].

PAN-MONTOJO, Juan Luis, “Juan Álvarez y Mendizábal (1790-1853): el burgués revolucionario”, in: Isabel Burdiel y Manuel Pérez Ledesma (coords.), Liberales, agitadores y conspiradores: biografías heterodoxas del siglo XIX, Madrid: Espasa, 2000, pp. 155-182.

POSA, Elena, "Actituds al voltant de la coeducació”, Papers: revista de sociología 9, Barcelona: Universidad Autónoma de Barcelona, 1978, pp. 55-72.

RUIZ, Julio, "Manuel Bartolomé Cossío y los comienzos de los estudios de Pedagogía en la Universidad de Madrid”, in: Julio Ruíz (ed.), Pedagogía y educación ante el siglo XXI, Madrid: Universidad Complutense de Madrid, 2005, pp. 117-142.

SCOTT, Joan W., "El eco de la fantasía: la historia y la construcción de la identidad", Ayer 62, Madrid: Marcial Pons, 2006, pp. 111-138.

SCOTT, Joan W., “Género: ¿Todavía una categoría útil para el análisis?”, La manzana de la discordia 6 , Cali: Universidad del Valle, 2011,pp. 95-101.

SUÁREZ, Luis: Crónica de la Sección Femenina y su tiempo, Madrid: Asociación Nueva Andadura, 1992. 
TAYLOR, Barbara, "Separations of Soul: Solitude, Biography, History", American Historical Review 114, Chicago: University of Chicago Press, 2009, pp. 640-651.

TUSELL, Javier, Historia de la democracia cristiana en España I. Los antecedentes. La CEDA y la II República, Madrid: Cuadernos para el diálogo, 1974.

VILLALAÍN, Pablo, Mujer y política, la participación de la mujer en las elecciones generales celebradas en Madrid durante la II república (1931-1936), Madrid: Instituto de la Mujer, 2000.

WALKOWITZ, Judith, City of Dreadful Delight: Narratives of Sexual Danger in Late-Victorian London, Londres: Virago, 1992.

\section{Breve información sobre el autor}

Universidad Autónoma de Madrid. Departamento de Historia Contemporánea.

Contratado FPU. Doctorando en Historia Contemporánea.

Su línea de investigación principal se centra en el estudio de cómo las conservadoras españolas resisten, redefinen y/o transgreden los modelos de género que se les pretende imponer.

Correo electrónico: alejandro.camino@uam.es 